\title{
AN APPLICATION OF MOTION CORRECTION METHODS TO THE ALIGNMENT PROBLEM IN NAVIGATION ${ }^{1}$
}

\author{
Boris I. Ananyev \\ Krasovskii Institute of Mathematics and Mechanics, \\ Ural Branch of Russian Academy of Sciences, Ekaterinburg, Russia, \\ abi@imm.uran.ru
}

\begin{abstract}
In this paper, we apply some motion correction methods to the alignment problem in navigation. This problem consists in matching two coordinate systems having the common origins. As a rule, one of the systems named as basic coordinate system is located at a ship or airplane. The dependent coordinate system belongs to another object (e.g. missile ) that starts from the ship. The problem is considered with incomplete information on state coordinates which can be measured with disturbances without statistical description.

Key words: Alignment problem, Motion correction, Incomplete information, Set-membership description of uncertainty.
\end{abstract}

\section{Introduction}

Alignment is the process whereby the orientation of the axes of an inertial navigation system is determined with respect to the reference axis system. The basic concept of aligning an inertial navigation system is quite simple and straightforward. However, there are many complications that make alignment both time consuming and complex. Consider a simulated transport ship-airplane system. Suppose that the base coordinate system (BCS) of the ship is correct. Let $\vec{\Omega}_{1}$ be the absolute angular velocity of the BCS in the motionless coordinate system $\eta_{1}, \eta_{2}, \eta_{3}$. The projection $\Omega_{1}^{2}$ on vertical $2_{1}$ equals zero. This system is shown on Fig. 1 . The axis $1_{1}$ is directed along the

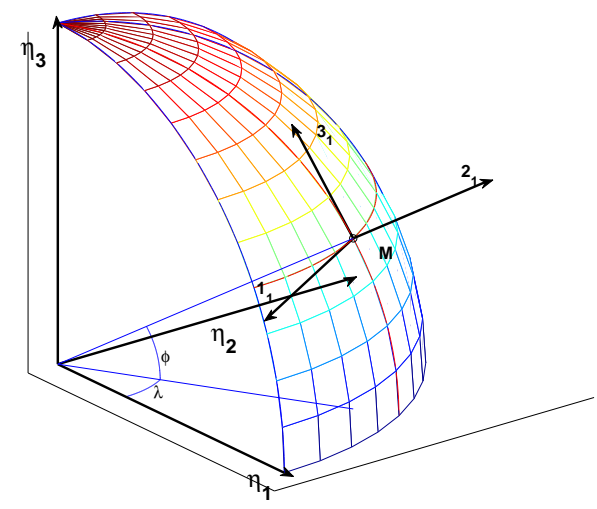

Figure 1. The section of Earth sphere and the base coordinate system.

parallel to the west. The axis $2_{1}$ is the local vertical. The axis $3_{1}$ is directed along the meridian to the north. The position of the dependent coordinate system (DCS) related to the airplane or

\footnotetext{
${ }^{1}$ The research was supported by Russian Science Foundation (RSF), project No. 16-11-10146.
} 
the missile with respect to the BCS is estimated by the Krylov angles. In Fig. 2, one can see the sequence of clockwise rotations: $\theta^{1}$ around axis $1, \theta^{3}$ around new axis 3 , and $\theta^{2}$ around new axis 2 coinciding now with $2_{1}$.

Thus, the transition of coordinates of a vector $\vec{f}$ in the DCS to new coordinates in the BCS is occurred by the formula $\vec{f}_{1}=\mathbf{M}(\theta) \vec{f}$, where the matrix of direction cosines is of the form

$$
\mathbf{M}(\theta)=\left(\begin{array}{ccc}
\cos \theta^{2} & 0 & -\sin \theta^{2} \\
0 & 1 & 0 \\
\sin \theta^{2} & 0 & \cos \theta^{2}
\end{array}\right) \cdot\left(\begin{array}{ccc}
\cos \theta^{3} & -\sin \theta^{3} & 0 \\
\sin \theta^{3} & \cos \theta^{3} & 0 \\
0 & 0 & 1
\end{array}\right) \cdot\left(\begin{array}{ccc}
1 & 0 & 0 \\
0 & \cos \theta^{1} & -\sin \theta^{1} \\
0 & \sin \theta^{1} & \cos \theta^{1}
\end{array}\right)=\left(m_{i j}\right) .
$$

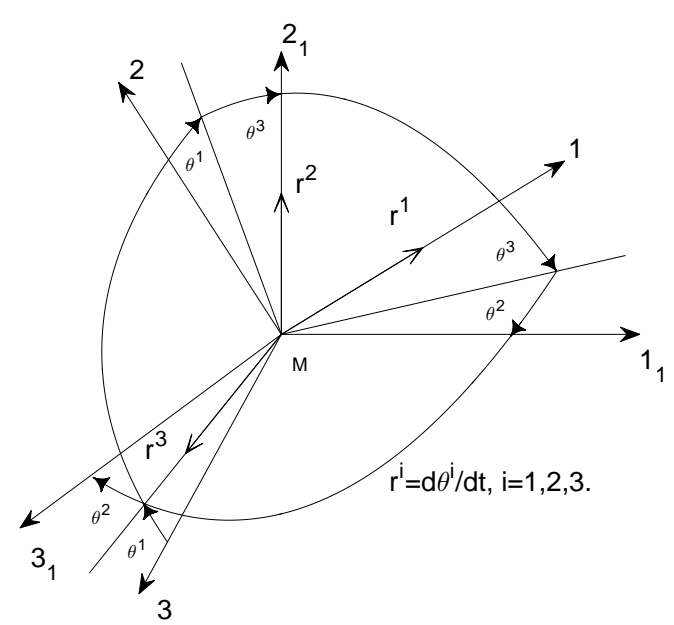

Figure 2. The sequence of clockwise rotations.

Projecting the equality $\vec{\omega}=\dot{\vec{\theta}}^{1}+\dot{\vec{\theta}}^{3}+\dot{\vec{\theta}}^{2}$ for the angular velocities on the axes of the DCS, we obtain the kinematic Krylov equations

$$
\dot{\theta}^{1}=\omega^{1}-\dot{\theta}^{2} \sin \theta^{3}, \quad \dot{\theta}^{2}=\left(\omega^{2} \cos \theta^{1}-\omega^{3} \sin \theta^{1}\right) / \cos \theta^{3}, \quad \dot{\theta}^{3}=\omega^{2} \sin \theta^{1}+\omega^{3} \cos \theta^{1},
$$

where $\omega^{i}$ are the projections of the relative angular velocity. These projections are related with the absolute velocities by the formulas

$$
\omega^{i}=\Omega^{i}-m_{1 i} \Omega_{1}^{1}-m_{3 i} \Omega_{1}^{3}+\varepsilon^{i}, \quad i \in 1: 3,
$$

where $\varepsilon^{i}$ are the projections of an uncertain drift.

For measurements, the differences of accelerometer readings in the DCS and BCS are used. These accelerometers are on the axes and gage the nongravity acceleration $\vec{a}=\vec{w}_{M}-\vec{g}$. Let $a^{i}$ be accelerometers readings in the DCS and $a_{1}^{i}$ be gage readings in the BCS. Therefore, the measurement equations are of the form

$$
\begin{gathered}
y^{1}=\left(m_{11}-1\right) a_{1}^{1}+m_{21} a_{1}^{2}+m_{31} a_{1}^{3}+w^{1}, \quad y^{2}=m_{12} a_{1}^{1}+\left(m_{22}-1\right) a_{1}^{2}+m_{32} a_{1}^{3}+w^{2}, \\
y^{3}=m_{13} a_{1}^{1}+m_{23} a_{1}^{2}+\left(m_{33}-1\right) a_{1}^{3}+w^{3},
\end{gathered}
$$

where $w^{i}$ are uncertain leavings of zero. About drifts $\varepsilon^{i}$ in $(0.2)$, the assumption is accepted that they are constant but unknown. Uncertain functions in relations (0.3) satisfy the integral inequalities

$$
\int_{0}^{T}\left(w^{i}\right)^{2} d t \leq \gamma_{i}^{2} T, \quad i \in 1: 3
$$


Let $\vec{i}_{1}, \vec{i}_{2}, \vec{i}_{3}$ be the unit direction vectors of the BCS. The velocity of point $M$ equals

$$
4 \vec{v}_{M}=\vec{\Omega}_{1} \times \vec{R}=\left|\begin{array}{ccc}
\vec{i}_{1} & \vec{i}_{2} & \vec{i}_{3} \\
\Omega_{1}^{1} & 0 & \Omega_{1}^{3} \\
0 & R & 0
\end{array}\right|,
$$

where $R$ is the radius of Earth. From here we find the projections of velocity on the BCS axes: $v_{1}^{1}=-R \Omega_{1}^{3}, v_{1}^{2}=0, v_{1}^{3}=R \Omega_{1}^{1}$. Computing the derivative of $\vec{v}_{M}$, we get the acceleration $\vec{w}_{M}=\widetilde{\vec{w}}_{M}+\vec{\Omega}_{1} \times \vec{v}_{M}$ in the form of the sum of relative and translation accelerations. So, the accelerometers readings in BCS are of the form:

$$
a_{1}^{1}=-R \dot{\Omega}_{1}^{3}, \quad a_{1}^{2}=g-v^{2} / R, \quad a_{1}^{3}=R \dot{\Omega}_{1}^{1},
$$

where $v$ is the velocity magnitude. As $R=6370 \mathrm{~km}$ and the velocity of the ship on water is no more than $20 \mathrm{~m} / \mathrm{c}$, we assume $a_{1}^{2}=g$.

Further we consider some approaches from motion correction for solving the alignment problem. This problem in inertial navigation was first in detail considered in [1]. Russian books devoted to this topic are [2-5]. The alignment problem was mostly solved in [1-5] by statistical methods with the help of Kalman filter or its modifications. On the other hand, in $[2,6]$ it was noted that the statistics of disturbances often happens incomplete or completely absent. Therefore, it is natural to use here the minimax methods from books $[7,8]$. Thus, all the disturbances in our paper are deterministic.

Consider only the case of small angular deviations (no more than several degrees). Equations (0.1) are replaced by the follwing ones:

$$
\begin{gathered}
\dot{\theta}^{1}=u^{1}+\varepsilon^{1}-\theta^{2} \Omega_{1}^{3}-\theta^{3} u^{2}, \quad \dot{\theta}^{2}=u^{2}+\varepsilon^{2}+\theta^{3} \Omega_{1}^{1}-\theta^{1} \Omega_{1}^{3}-\theta^{1} u^{3}, \\
\dot{\theta}^{3}=u^{3}+\varepsilon^{3}+\theta^{2} \Omega_{1}^{1}+\theta^{1} u^{2} .
\end{gathered}
$$

Here, $u^{i}=\Omega^{i}-\Omega_{1}^{i}, i \in 1: 3$. In the linear approximation, the differences of accelerometer readings in $(0.3)$ are equal to

$$
y^{1}=g \theta^{3}+a_{1}^{3} \theta^{2}+w^{1}, \quad y^{2}=-a_{1}^{1} \theta^{3}+a_{1}^{3} \theta^{1}+w^{2}, \quad y^{3}=-a_{1}^{1} \theta^{2}-g \theta^{1}+w^{3} .
$$

Equations (0.6) contain the multiplications of controls and state variables, but, in the case of small angles and angular velocities these terms may be neglected. In the specific case of movement on the equator under condition $\theta^{1}=\theta^{2} \equiv 0$, we assume $\theta=\theta^{3}$ as shown on Fig. 3. The angular velocity $\Omega_{1}=\Omega_{1}^{3} \neq 0$ under given movement and the rest projections of absolute angular velocity are equal to zero. We have

$$
\dot{\theta}=u+\varepsilon, \quad \dot{\varepsilon}=0, \quad y=g \theta+w,
$$

where the first equation from (0.7) is taken as the output.

\section{Set-membership background}

So, we consider a determinate $n$-dimensional linear system of the form

$$
\dot{x}(t)=A(t) x+B(t) u+C(t) v, \quad t \in[0, T],
$$

assuming that the initial state $x_{0}$ of system (1.1) is completely unknown, the matrices $A(t), B(t), C(t)$, and $G(t)$ below are continuous. In comparison with equations (0.6), the term with disturbance $v$ in 


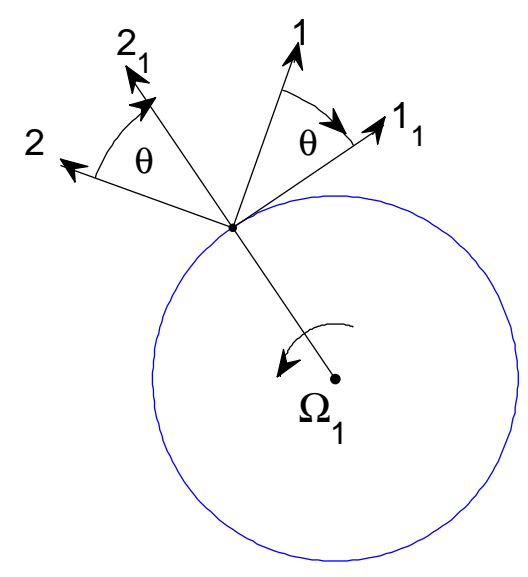

Figure 3. System deviation in the simple model.

the system is added here. It corresponds to the case when drifts are not constants. The unknown function $v(\cdot)$ and the disturbance $w(\cdot)$ in the $m$-dimensional equation of measurement

$$
y(t)=G(t) x(t)+w(t)
$$

are bounded by the constraint

$$
\int_{0}^{T}\left(|v(t)|_{Q(t)}^{2}+|w(t)|_{R(t)}^{2}\right) d t \leq 1
$$

where the symbol $|x|_{P}^{2}$ equals $x^{\prime} P x$, prime ' means the transposition, $Q(t), R(t)$ are symmetrical, positive-defined, and continuous matrices having suitable dimension. Constraint (1.3) involves that the elements of vector functions $v(\cdot)$ and $w(\cdot)$ belong to the space $L_{2}[0, T]$. We need the following

Assumption 1. The system (1.1), (1.2) under $u \equiv 0, v \equiv 0, w \equiv 0$ is completely observable [7] on any subinterval $[s, \tau] \subset[0, T]$.

Assumption 1 means that the vector $x(s)$ can be uniquely restored from the signal observed on $[s, \tau]$ if the disturbances are absent. Moreover, Assumption 1 holds if and only if

$$
\int_{s}^{\tau} X^{\prime}(t, s) G^{\prime}(t) G(t) X(t, s) d t>0
$$

where $X(t, s)$ is the fundamental matrix of system (1.1).

We use piecewise-constant functions $u(t)$, for which

$$
u(t) \in P \subset \mathbb{R}^{p},
$$

where $P$ is a compact convex set. Constraint (1.4) is more realistic than integral constraints in [9]. The aim of the control is to minimize the terminal function $|D x(T)|$, where $|\cdot|$ is the Euclidean norm and $D \in \mathbb{R}^{d \times n}$ is a matrix. The choice of uncertain parameters $\left\{x_{0}, v(\cdot), w(\cdot)\right\}$ may impede the minimization. 


\subsection{Informational and compatible sets}

At first, let us consider a set-membership estimation scheme for system (1.1), (1.2) under constraint (1.3).

Definition 1. A set $\mathbf{X}(t, y, u) \subset \mathbb{R}^{n}$ is said to be the informational if it consists of all vectors $x=x(t)$, which may realize in system (1.1), (1.2) with given signal $y(\tau), 0 \leq \tau \leq t$, the control $u(\tau)$, and some disturbances satisfying constraint (1.3).

To describe the informational set, we introduce the Bellman function

$$
V(t, x)=\inf _{v(\cdot)}\left\{\int_{0}^{t}\left(|v(s)|_{Q(s)}^{2}+|y(s)-G(s) x(s)|_{R(s)}^{2}\right) d s\right\}, \quad x(t)=x .
$$

The Bellman equation for $V(t, x)$ is of the form:

$$
V_{t}=\min _{v}\left\{-(A(t) x+B(t) u(t)+C(t) v)^{\prime} V_{x}+|v|_{Q(t)}^{2}+|y(t)-G(t) x|_{R(t)}^{2}\right\}, \quad V(0, x)=0 .
$$

If the solution of equation (1.5) in any sense is found, the informational set $\mathbf{X}(t, y, u)$ is written as the inequality $\mathbf{X}(t, y, u)=\{x: V(t, x) \leq 1\}$. Let us seek a solution of equation (1.5) in the form

$$
V(t, x)=|x|_{P(t)}^{2}-2 x^{\prime} d(t)+g(t),
$$

where $P(t)$ is a positive definite and continuously differentiable matrix, $d(t)$ and $g(t)$ are a continuously differentiable vector function and a function respectively. Substituting (1.6) into (1.5), we get

$$
\begin{aligned}
|x|_{\dot{P}(t)}^{2}-2 x^{\prime} \dot{d}(t)+ & \dot{g}(t)=|y(t)-G(t) x|_{R(t)}^{2}-|P(t) x-d(t)|_{C(t) Q^{-1}(t) C^{\prime}(t)}^{2}- \\
& -2(A(t) x+B(t) u(t))^{\prime}(P(t) x-d(t)) .
\end{aligned}
$$

Therefore, the parameters of (1.6) must satisfy the equations

$$
\begin{gathered}
\dot{P}(t)=G^{\prime}(t) R(t) G(t)-P(t) C(t) Q^{-1}(t) C^{\prime}(t) P(t)-A^{\prime}(t) P(t)-P(t) A(t), \quad P(0)=0, \\
\dot{d}(t)=G^{\prime}(t) R(t) y(t)-\left(P(t) C(t) Q^{-1}(t) C^{\prime}(t)+A^{\prime}(t)\right) d(t)+P(t) B(t) u(t), \quad d(0)=0, \\
\dot{g}(t)=|y(t)|_{R(t)}^{2}-|d(t)|_{C(t) Q^{-1}(t) C^{\prime}(t)}^{2}+2 d^{\prime}(t) B(t) u(t), \quad g(0)=0 .
\end{gathered}
$$

It is known [10] that the matrix $P(t)$ is non-singular for any $t>0$ under Assumption 1 . Then the ellipsoid (informational set)

$$
\mathbf{X}(t, y, u)=\left\{x \in \mathbb{R}^{n}: V(t, x)=|x|_{P(t)}^{2}-2 x^{\prime} d(t)+g(t)=|x-\hat{x}(t)|_{P(t)}^{2}+h(t) \leq 1\right\}
$$

is bounded for any $t>0$ with the center $\hat{x}(t)=P^{-1}(t) d(t)$ and the function $h(t)=g(t)-|d(t)|_{P^{-1}(t)}^{2}$. Differentiating the $\hat{x}(t)$ and $h(t)$, we obtain the equations

$$
\begin{gathered}
\dot{\hat{x}}(t)=A(t) \hat{x}(t)+B(t) u(t)+P^{-1}(t) G^{\prime}(t) R(t)(y(t)-G(t) \hat{x}(t)), \\
\dot{h}(t)=|y(t)-G(t) \hat{x}(t)|_{R(t)}^{2} .
\end{gathered}
$$

Let us introduce the function

$$
f(t)=y(t)-G(t) \hat{x}(t), \quad t \in(0, T],
$$

that is similar to the innovation process in theory of Kalman filtering [10]. 
Lemma 1. Function (1.10) does not depend on the control $u(t)$, belongs to the space $L_{2}^{m}[0, T]$, and we have

$$
h(t)=\int_{0}^{t}|y(s)-G(s) \hat{x}(s)|_{R(s)}^{2} d s \leq 1, \quad t \in[0, T] .
$$

On the other hand, let the instant $\tau \in(0, T)$ and $f(\cdot)$ be any function from $L_{2}^{m}[\tau, T]$ with

$$
\int_{\tau}^{T}|f(s)|_{R(s)}^{2} d s \leq 1-h(\tau)
$$

Then we obtain

$$
\mathbf{X}(t, y, u)=\left\{x \in \mathbb{R}^{n}:|x-\check{x}(t)|_{P(t)}^{2}+h(t) \leq 1\right\}, \quad t \in[\tau, T]
$$

where

$$
\begin{gathered}
\dot{\check{x}}(t)=A(t) \check{x}(t)+B(t) u(t)+P^{-1}(t) G^{\prime}(t) R(t) f(t), \\
\check{x}(\tau)=\hat{x}(\tau) ; \quad h(t)=h(\tau)+\int_{\tau}^{t}|f(s)|_{R(s)}^{2} d s .
\end{gathered}
$$

Here, we set $y(t)=f(t)+G(t) \check{x}(t), t \in[\tau, T]$.

P r o o f. As $0 \leq h(t) \leq g(t)$ and $g(0)=0$, we conclude that $h(0)=0$. From (1.8) and (1.9) we obtain the formula for $h(t)$. The signal $y(\cdot)$ may realize in system $(1.1),(1.2)$ on $[\tau, t], t \in(\tau, T]$, under closed-loop disturbance $v(s)=Q^{-1}(s) C^{\prime}(s)(P(s) x(s)-d(s))$ that gives minimum to the functional according to (1.5), (1.6), and (1.7) with any final state $x(t)=x \in \mathbf{X}(t, y, u)$. As the formulas for $\check{x}(t)$ coincide with (1.9), formula (1.11) holds.

From now on, the narrowings of a measurable vector-function $x(s), s \in[0, T]$, on intervals $[0, t]$ and $[t, T]$ are denoted by $x^{t}(\cdot)$ and $x_{t}(\cdot)$ respectively. The narrowing on $[t, s]$ is denoted by $x_{t}^{s}(\cdot)$. Let the dimension of the disturbance $v$ be equal $q$.

Definition 2. A set $\mathbf{V}(t, y, u) \subset \mathbb{R}^{n} \times L_{2}^{q}[t, T] \times L_{2}^{m}[t, T]$ is said to be the compatible if it consists of all triples $\left\{\left(x(t), v_{t}(\cdot), w_{t}(\cdot)\right)\right\}$, for which there exist functions $(v(\cdot), w(\cdot))$ satisfying $(1.3)$ such that output $(1.2)$ on $[0, t]$ with final state $x=x(t)$ almost everywhere coincides with the given signal $y^{t}(\cdot)$.

Note that the sets $\mathbf{X}(t, y, u)$ and $\mathbf{V}(t, y, u)$ depend only on $y^{t}(\cdot)$ and $u^{t}(\cdot)$. Suppose that we have the compatible set $\mathbf{V}(t, y, u)$, and on the interval $[t, s]$ a signal $y_{t}^{s}(\cdot)$ and a control $u_{t}^{s}(\cdot)$ are realized. Similarly to Definitions 1 and 2, we can define the sets $\mathbf{X}\left(s, y_{t}^{s}, u_{t}^{s} \mid \mathbf{V}(t, y, u)\right)$ and $\mathbf{V}\left(s, y_{t}^{s}, u_{t}^{s} \mid\right.$ $\mathbf{V}(t, y, u))$. The following assertion seems to be obvious.

Lemma 2. The relation between compatible and information sets is given by the equality $\mathbf{X}(t, y$, $u)=\operatorname{proj}_{\mathbb{R}^{n}} \mathbf{V}(t, y, u)$. The compatible set is described by the formula

$$
\mathbf{V}(t, y, u)=\left\{\left(x, v_{t}, w_{t}\right): \int_{t}^{T}\left(\left|v_{t}(s)\right|_{Q(s)}^{2}+\left|w_{t}(s)\right|_{R(s)}\right) d s+V(t, x) \leq 1\right\},
$$

where $V(t, x)$ is defined in (1.6) or (1.8). Under Assumption 1, set (1.12) is weakly compact in the space $\mathbb{R}^{n} \times L_{2}^{q}[t, T] \times L_{2}^{m}[t, T]$ when $t \in(0, T)$. Moreover, compatible sets posses the semigroup property: $\mathbf{V}\left(s, y_{t}^{s}, u_{t}^{s} \mid \mathbf{V}(t, y, u)\right)=\mathbf{V}(s, y, u)$, where $0<t<s \leq T$. As a consequence, we have $\mathbf{X}\left(s, y_{t}^{s}, u_{t}^{s} \mid \mathbf{V}(t, y, u)\right)=\mathbf{X}(s, y, u)$.

The final reachable set of system (1.1) from the compatible set $V(t, y, u)$ is denoted further by $\mathbf{X}_{T}\left(u_{t} \mid \mathbf{V}(t, y, u)\right)$. This set consists of all vectors $x(T)$ under searching in (1.12) for the set $V(t, y, u)$ with $w_{t}=0$. 


\section{Problems formulation}

Let $\lambda: 0<t_{1}<\cdots<t_{N+1}=T$ be a partition of the interval $[0, T]$. The times $t_{i}$ are called the instants of control correction. It is easily seen that the compatible set $\mathbf{V}(t, y, u)$ depends only on the pair $(\hat{x}(t), h(t))$ which is called the position at the instant $t$. The transition between two adjacent positions $\left(\hat{x}\left(t_{i}\right), h\left(t_{i}\right)\right)$ and $\left(\hat{x}\left(t_{i+1}\right), h\left(t_{i+1}\right)\right)$ depends on the control $u_{i}(\cdot)$ and the innovation function $f_{i}(\cdot)$ on the interval $\left[t_{i}, t_{i+1}\right)$ according to Lemma 1 . Consider two problems.

Problem 1. Find a piecewise-constant control $u^{*}(t)\left(u^{*}(t)=u_{i}^{*}\right.$ on $\left.\left[t_{i}, t_{i+1}\right), i \in 1: N\right)$ that gives the value

$$
J^{*}=\min _{u_{1} \in P} \max _{f_{1}(\cdot)} \ldots \min _{u_{N} \in P} \max _{f_{N}(\cdot)} \max _{x \in \mathbf{X}_{T}\left(u_{N} \mid \mathbf{V}\left(t_{N}, y, u\right)\right)}|D x|,
$$

where

$$
\sum_{i=1}^{N} \int_{t_{i}}^{t_{i+1}}\left|f_{i}(s)\right|_{R(s)}^{2} d s \leq 1-h\left(t_{1}\right)
$$

Remark 1. As equations (1.1), (1.2) are linear, we have $\mathbf{X}(t, y, u)=z(t)+\mathbf{X}(t, \tilde{y}, 0)$, where $\tilde{y}(t)=y(t)-G(t) z(t)$ and

$$
\dot{z}(t)=A(t) z(t)+B(t) u(t), \quad z(0)=0 .
$$

Similarly, we have $\mathbf{V}(t, y, u)=(z(t), 0,0)+\mathbf{V}(t, \tilde{y}, 0)$. From now on, we write the sets with $\tilde{y}(\cdot)$ and $u(\cdot)=0$ as $\mathbf{X}(t, \tilde{y})$ and $\mathbf{V}(t, \tilde{y})$, respectively. Therefore, $\mathbf{X}_{T}\left(u_{t} \mid \mathbf{V}(t, y, u)\right)=z(T)+\mathbf{X}_{T}(0 \mid \mathbf{V}(t, \tilde{y}))$ and value (2.1) may be rewritten as

$$
J^{*}=\min _{u_{1} \in P} \max _{f_{1}(\cdot)} \ldots \min _{u_{N} \in P} \max _{f_{N}(\cdot)} \max _{x \in \mathbf{X}_{T}\left(0 \mid \mathbf{V}\left(t_{N}, \tilde{y}\right)\right)}|D(z(T)+x)| .
$$

Remark 2. We obtain as a fact that controls $u_{i}$ in (2.1) and (2.3) depend on the positions $\left(\hat{x}\left(t_{i}\right), h\left(t_{i}\right)\right)$. Problem 1 may be generalized if we seek non-constant functions $u_{i}(\cdot)$ on the interval $\left[t_{i}, t_{i+1}\right)$.

Problem 2. At the any instant $t_{i}, i \in 1: N$, we find open loop minimax control $u_{i}^{T *}(\cdot)$ that give a solution of the problem:

$$
\max _{f_{i}(\cdot)} \max _{x \in \mathbf{X}_{T}\left(u_{i} \mid \mathbf{V}\left(t_{i}, y, u\right)\right)}|D x| \rightarrow \min _{u_{i}(t) \in P}=j_{i}(y),
$$

where

$$
\int_{t_{i}}^{T}\left|f_{i}(s)\right|_{R(s)}^{2} d s \leq 1-h\left(t_{i}\right)
$$

and do one-step forecasting

$$
J_{i}\left(y, u_{i}\right)=\max _{f_{i}(\cdot)} j_{i+1}(y),
$$

where

$$
\int_{t_{i}}^{t_{i+1}}\left|f_{i}(s)\right|_{R(s)}^{2} d s \leq 1-h\left(t_{i}\right) .
$$

If $J_{i}\left(y, u_{i}^{T *}\right)<j_{i}(y)$ we keep the control $u_{i}^{T *}$ on the interval $\left[t_{i}, t_{i+1}\right]$. Otherwise, we pass to the control $u_{i}^{i+1 *}$ that minimizes value (2.5). Of course, the controls may be not unique. If so, we choose any minimizers. 


\section{Minimax solutions}

For brevity we denote $\hat{x}\left(t_{i}\right)=\hat{x}_{i}$ and $h\left(t_{i}\right)=h_{i}$. Introduce the function of next losses

$$
W_{i}\left(\hat{x}_{i}, h_{i}\right)=\min _{u_{i} \in P} \max _{f_{i}(\cdot)} \ldots \min _{u_{N} \in P} \max _{f_{N}(\cdot)} \max _{x \in \mathbf{X}_{T}\left(u_{t_{N}} \mid \mathbf{V}\left(t_{N}, y, u\right)\right)}|D x|
$$

where

$$
\sum_{j=i}^{N} \int_{t_{j}}^{t_{j+1}}\left|f_{j}(s)\right|_{R(s)}^{2} d s \leq 1-h_{i}
$$

for Problem 1. It is easily seen that the functions $W_{i}\left(\hat{x}_{i}, h_{i}\right)$ satisfy the following recurrent relations

$$
W_{i}\left(\hat{x}_{i}, h_{i}\right)=\min _{u_{i} \in P} \max _{f_{i}(\cdot)} W_{i+1}\left(\hat{x}_{i+1}, h_{i+1}\right),
$$

where

$$
\int_{t_{i}}^{t_{i+1}}\left|f_{i}(s)\right|_{R(s)}^{2} d s \leq 1-h_{i}
$$

Relations (3.1) have the boundary condition

$$
W_{N+1}(\hat{x}(T), h(T))=\max _{|x-\hat{x}(T)|_{P(T)}^{2} \leq 1-h(T)}|D x|=\max _{|l| \leq 1}\left\{l^{\prime} D \hat{x}(T)+(1-h(T))^{1 / 2}\left|D^{\prime} l\right|_{P^{-1}(T)}\right\} .
$$

Consider the last stage of relations (3.1) when $i=N$. Using boundary condition, we obtain

$$
\begin{aligned}
W_{N}\left(\hat{x}_{N}, h_{N}\right)=\max _{|l| \leq 1}\left\{r\left(l ; t_{N}\right) \hat{x}_{N}\right. & +\min _{u \in P} \int_{t_{N}}^{T} r(l ; s) B(s) d s u+\left(( 1 - h _ { N } ) \left(\lambda\left(t_{N}\right)\left(1-|l|^{2}\right)\right.\right. \\
& \left.\left.\left.+\left|D^{\prime} l\right|_{P\left(T, t_{N}\right)}^{2}\right)\right)^{1 / 2}\right\}
\end{aligned}
$$

where

$$
\begin{gathered}
r(l ; s)=l^{\prime} D X(T, s), \quad \partial P(t, s) / \partial t=A(t) P(t, s)+P(t, s) A^{\prime}(t)+C(t) Q^{-1}(t) C^{\prime}(t), \\
P(s, s)=P^{-1}(s), \quad \lambda(s)=\max _{|l| \leq 1}\left|D^{\prime} l\right|_{P(T, s)}^{2} .
\end{gathered}
$$

Here, the term with integral must be replaced on

$$
\int_{t_{N}}^{T} \min _{u \in P} r(l ; s) B(s) u d s
$$

if the control is not piecewise-constant. Let us explain the formula for $W_{N}\left(\hat{x}_{N}, h_{N}\right)$. It is obtained with the help of elementary equality

$$
\max _{k \in\left[0,1-h_{N}\right]}\left\{k^{1 / 2} A+\left(1-h_{N}-k\right)^{1 / 2} B\right\}=\left(1-h_{N}\right)^{1 / 2}\left(A^{2}+B^{2}\right)^{1 / 2},
$$

where $A \geq 0, B \geq 0$, and the maximum is achieved at $r^{*}=\left(1-h_{N}\right) A^{2}\left(A^{2}+B^{2}\right)^{-1 / 2}$. Besides, the optimization over $f(\cdot)$ is fulfilled under the constraint $\int_{t_{N}}^{T}|f(s)|_{R(s)}^{2} d s=k$. If $\lambda(s)$ is the maximal eigenvalue of the matrix $\operatorname{DP}(T, s) D^{\prime}$, we use the fact that conc $|l|_{Q}$ on unite ball is equal to $\left(\lambda_{\max }\left(1-|l|^{2}\right)+|l|_{Q}^{2}\right)^{1 / 2}$, see $[7]$. Hereinafter, the symbol conc $\varphi(l)$ means a minimal concave function majorizing $\varphi(l)$ on unite ball. At last, we apply the minimax theorem.

Continuing calculations on the subsequent stages, we come to the conclusion. 
Theorem 1 (Conditions of the optimality in Problem 1). On the stage $i$, we have

$$
\begin{gathered}
W_{i}\left(\hat{x}_{i}, h_{i}\right)=\max _{|l| \leq 1}\left\{r\left(l ; t_{i}\right) \hat{x}_{i}+\min _{u \in P} \int_{t_{i}}^{t_{i+1}} r(l ; s) B(s) d s u+\varphi_{i}(l)\right\} \text {, where } \\
\varphi_{i}(l)=\operatorname{conc}\left\{\min _{u \in P} \int_{t_{i+1}}^{t_{i+2}} r(l ; s) B(s) u+\max _{f_{i}(\cdot)}\left\{\int_{t_{i}}^{t_{i+1}} r(l ; s) P^{-1}(s) G^{\prime}(s) R(s) f_{i}(s) d s\right.\right. \\
\left.\left.+\varphi_{i+1}(l)\right\}\right\}, \quad i \in 1: N-1 .
\end{gathered}
$$

Here

$$
\int_{t_{i}}^{t_{i+1}}\left|f_{i}(s)\right|_{R(s)}^{2} d s \leq 1-h_{i} .
$$

The optimal controls necessarily satisfy the relation

$$
\begin{gathered}
\int_{t_{i}}^{t_{i+1}} r\left(l^{*} ; s\right) B(s) d s u_{i}^{*}=\min _{u \in P} \int_{t_{i}}^{t_{i+1}} r\left(l^{*} ; s\right) B(s) d s u \text { or } \int_{t_{i}}^{t_{i+1}} r\left(l^{*} ; s\right) B(s) u_{i}^{*}(s) d s \\
\quad=\int_{t_{i}}^{t_{i+1}} \min _{u \in P} r\left(l^{*} ; s\right) B(s) u d s \quad \text { if the control is not piecewise-constant }
\end{gathered}
$$

where $l^{*}$ is a maximizer in problem (3.3).

P r o o f. For the first two stages, we have

$$
\begin{gathered}
\varphi_{N}(l)=\left(\left(1-h_{N}\right)\left(\lambda\left(t_{N}\right)\left(1-|l|^{2}\right)+\left|D^{\prime} l\right|_{P\left(T, t_{N}\right)}^{2}\right)\right)^{1 / 2}, \\
\varphi_{N-1}(l)=\operatorname{conc}\left\{\min _{u \in P} \int_{t_{N}}^{T} r(l ; s) B(s) d s u+\max _{f_{N-1}(\cdot)}\left\{\int_{t_{N-1}}^{t_{N}} r(l ; s) P^{-1}(s) G^{\prime}(s) R(s) f_{N-1}(s) d s\right.\right. \\
\left.\left.+\varphi_{N}(l)\right\}\right\}=\operatorname{conc}\left\{\min _{u \in P} \int_{t_{N}}^{T} r(l ; s) B(s) d s u+\left(\left(1-h_{N-1}\right)\left(\lambda\left(t_{N}\right)\left(1-|l|^{2}\right)+\left|D^{\prime} l\right|_{P\left(T, t_{N-1}\right)}^{2}\right)\right)^{1 / 2}\right\} .
\end{gathered}
$$

For derivation of the last relation, we use the same reasoning as for $W_{N}\left(\hat{x}_{N}, h_{N}\right)$. The subsequent considerations are obtained by induction with the help of the minimax theorem.

To solve Problem 2, we need to calculate values (2.4), (2.5). Doing as above we get

$$
\begin{gathered}
j_{i}(y)=\max _{|l| \leq 1}\left\{r\left(l ; t_{i}\right) \hat{x}_{i}+\int_{t_{i}}^{T} \min _{u \in P} r(l ; s) B(s) u d s+\left(( 1 - h _ { i } ) \left(\lambda\left(t_{i}\right)\left(1-|l|^{2}\right)\right.\right.\right. \\
\left.\left.\left.+\left|D^{\prime} l\right|_{P\left(T, t_{i}\right)}^{2}\right)\right)^{1 / 2}\right\}, \\
J_{i}\left(y, u_{i}\right)=\max _{f_{i}(\cdot)} j_{i+1}(y)=\max _{|l| \leq 1}\left\{r\left(l ; t_{i}\right) \hat{x}_{i}+\int_{t_{i}}^{t_{i+1}} r(l ; s) B(s) u_{i}(s) d s\right. \\
\left.+\int_{t_{i+1}}^{T} \min _{u \in P} r(l ; s) B(s) u d s+\left(\left(1-h_{i}\right)\left(\lambda\left(t_{i+1}\right)\left(1-|l|^{2}\right)+\left|D^{\prime} l\right|_{P\left(T, t_{i}\right)}^{2}\right)\right)^{1 / 2}\right\} .
\end{gathered}
$$

Theorem 2 (Properties of controls in Problem 2). The control procedure in Problem 2 begins from $i=1$ and leads to a sequence of positions, where $j_{1}(y) \geq j_{2}(y) \geq \cdots \geq j_{N}(y)$. 
P r o o f. Let us compare the values $j_{i}(y)$ and $j_{i+1}(y)$. If $J_{i}\left(y, u_{i}^{T *}\right)<j_{i}(y)$, we get $j_{i}(y)>$ $j_{i+1}(y)$. Otherwise, we use the control $u_{i}^{i+1 *}$ that minimizes the value $J_{i}\left(y, u_{i}\right)$. Therefore,

$$
\begin{gathered}
\min _{u_{i}(\cdot)} J_{i}\left(y, u_{i}\right)=\max _{|l| \leq 1}\left\{r\left(l ; t_{i}\right) \hat{x}_{i}+\int_{t_{i}}^{t_{i+1}} \min _{u \in P} r(l ; s) B(s) u d s\right. \\
\left.+\operatorname{conc}\left\{\int_{t_{i+1}}^{T} \min _{u \in P} r(l ; s) B(s) u d s+\left(\left(1-h_{i}\right)\left(\lambda\left(t_{i+1}\right)\left(1-|l|^{2}\right)+\left|D^{\prime} l\right|_{P\left(T, t_{i}\right)}^{2}\right)\right)^{1 / 2}\right\}\right\} \leq j_{i}(y),
\end{gathered}
$$

as $\lambda\left(t_{i+1}\right) \leq \lambda\left(t_{i}\right)$. The last inequality implies the relation

$$
\partial P(T, s) / \partial s=-X(T, s) P^{-1}(s) G^{\prime}(s) R(s) G(s) P^{-1}(s) X^{\prime}(T, s),
$$

whence the norm of the matrix $P(T, s)$ decreases on s.

Remark 3. The procedure of calculation of optimal controls in Problem 1 is more difficult than in Problem 2. But we can simplify it if by a slight increase of the function of future losses. Namely, we have $W_{i}\left(\hat{x}_{i}, h_{i}\right) \leq j_{i}(y)$. This inequality follows by induction from relations (3.3)-(3.5). One can find the controls in this simplified procedure by formulas (3.4).

To illustrate the different approaches to optimal control, consider a simple

Example. Given the one-dimensional system $\dot{x}=u+v, 0 \leq t \leq 3$, with the measurement $y(t)=x(t)+w$ and the constraints

$$
x_{0}^{2}+\int_{0}^{3}\left(v^{2}(t)+w^{2}(t)\right) d t \leq 1
$$

$|u| \leq 1 / 2$, we suppose $y(t) \equiv 1$ on $[0,3]$. Let $t_{1}=1, t_{2}=2$ be two correction instants. Here, we add the limitation on initial state for simplicity.

We have $P \equiv 1, \hat{x}(t)=1-e^{-t}, h(t)=\left(1-e^{-2 t}\right) / 2$ on $[0,3]$ under $u \equiv 0$, as follows from (1.7), $P(T, s)=4-s$. The unknown real movement $x(t) \equiv 1$ under $u \equiv 0$. Formula (3.3) gives $W_{1}\left(\hat{x}_{1}, h_{1}\right)=1.0655$ and optimal control on $[1,2]$ equals $u_{1}=-0.5$. Here, the choice of control is not unique. At the next stage $W_{2}\left(\hat{x}_{2}, h_{2}\right)=1.0091$ and the optimal control on $[2,3]$ equals $u_{2}=-\hat{x}_{2}=e^{-2}-1 / 2=-0.3647$. In Problem 2, we have $j_{1}(y)=1.3050$ and we obtain the same sequence of optimal controls. At last, consider the partition of $[1,3]$ with step $0.25, N=8$, and we use the procedure of Remark 3. This procedure leads us to the sequence of control $u_{i}=-0.5$ at each step. The final value of the functional equals 0.7577 .

\section{Numerical simulation of alignment process}

We restrict ourself by the consideration of the simple case of system (0.8) and the procedure of Remark 3. The qualitative sense does not change in the common case.

The following data are used: $\left|\theta^{i}\right| \leq 3 \mathrm{grad},\left|\varepsilon^{i}\right| \leq 0.1 \mathrm{grad} / \mathrm{sec},\left|u_{i}\right| \leq 0.1 \mathrm{rad} / \mathrm{sec}, T=100$ sec. In integral constraint (0.4) the constants are $\gamma_{i}=0.1 \mathrm{~m} / \mathrm{sec}^{2}$. The signal is given by $w(t)=$ $\sin (t) / \sqrt{55}$. The alignment process is shown on the figures.

\section{Conclusion}

In this paper, we consider the application of motion correction methods to the alignment problem in inertial navigation. We use the deterministic approach with set-membership description of uncertainty. The Theorems 1, 2 and the procedure in Remark 3 are new. The investigation of the influence of ship movement on the accuracy of alignment was not performed. It will be done in subsequent papers. 


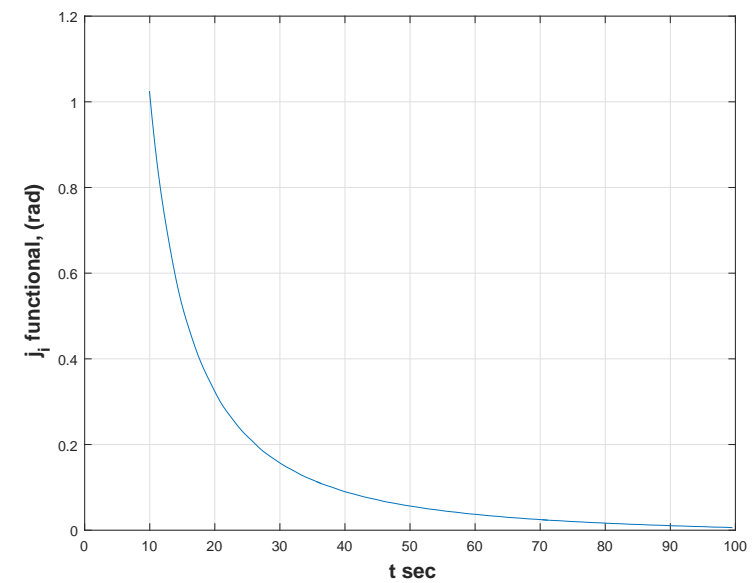

Figure 4. Alteration of the functional in the simple model.

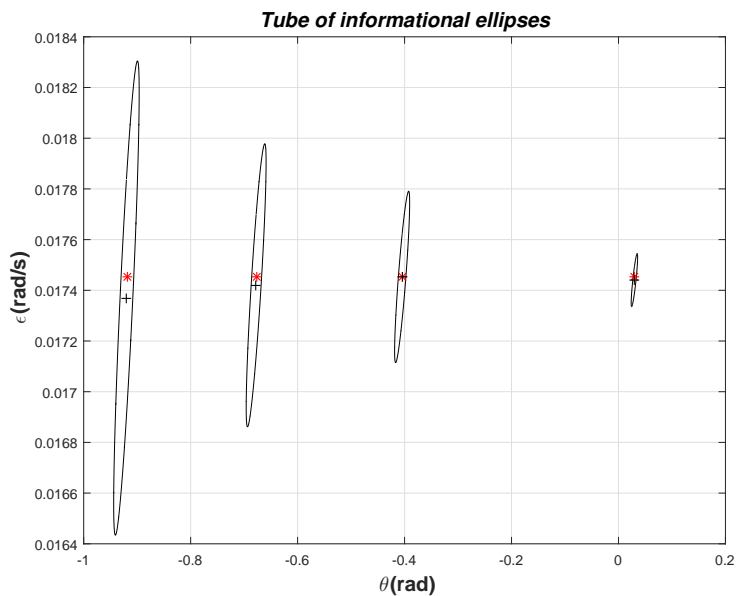

Figure 5. Informational ellipses at the instants $t=44,58,72$, and 100 .

\section{REFERENCES}

1. Lipton A.H. Alignment of Inertial Systems on a Moving Base. Washington D.C.: NASA, 1967. 178 p.

2. Boguslavski I.A. Applied Problems of Filtering and Control. M.: Nauka, 1983. 314 p. [in Russian]

3. Bromberg P.V. The Theory of Inertial Navigation Systems. M.: Nauka, 1979. 245 p. [in Russian]

4. Klimov D.M. Inertial Navigation on the Sea. M.: Nauka, 1984. 211 p. [in Russian]

5. Parusnikov N.A., Morozov V.M, Borzov V.I. Correction Problem in Inertial Navigation. M.: MGU, 1982. 256 p. [in Russian]

6. Bachshiyan B.Ts., Nazirov R.R., Eliyasberg P.E. Determination and Correction of Motion. M.: Nauka, 1980. 402 p. [in Russian]

7. Kurzhanski A.B. Control and Observation under Conditions of Uncertainty. M.: Nauka, 1977. 392 p. [in Russian]

8. Krasovskii N.N. and Subbotin A.I. Game-Theoretical Control Problems. Springer-Verlag, New York, 1988. $517 \mathrm{p}$.

9. Ananyev B.I. and Gredasova N.V. The Alignment Problem of Inertial Systems and Motion Correction Procedure // Bulletin of Buryatian State University, 2011. No. 9. P. 203-208. old.bsu.ru/content/pages2/1074/2011/AnanevBI.pdf [in Russian]

10. Liptser R.Sh. and Shiryayev A.N. Statistics of Random Processes, V.1 General Theory, V.2 Applications, Springer-Verlag, New York, 2000. 